\title{
DISEÑO DE UNA METODOLOGÍA PARA LA ESCRITURA DE PROYECTOS DOCUMENTALES EN EL AULA
}

René Palomino Rodríguez - Yulenis Stella Caballero Mier - Frank Alexander Rodríguez 


\section{DESIGN OFAMETHODOLOGYTO WRITE DOCUMENTARYPROJECTS INTHE CLASSROOM}

\begin{abstract}
RESUMEN
El presente artículo expone la pertinencia del diseño de una metodología para la escritura y el desarrollo de proyectos documentales en el aula, en el marco de la profundización documental de la carrera de pregrado de Artes audiovisuales de la Universidad Autónoma de Bucaramanga. Teniendo en cuenta las prácticas en el aula de los profesores que intervienen en las asignaturas de realización de cine documental y haciendo una revisión del estado del arte sobre los imaginarios y las distintas formas de la escritura para documentales, se identifican ciertos problemas metodológicos en relación con la escritura, recurrentes en el proceso de diseño y realización de proyectos documentales. Por este motivo, el objetivo de la investigación en el aula es establecer una estrategia metodológica que contribuya al proceso creativo para el diseño y la escritura de obras documentales en el aula.
\end{abstract}

Palabras clave: cine, documental, guion, metodología, géneros textuales.

\begin{abstract}
This article depicts the relevance of the design in a methodology for the writing and development of documentary projects in the classroom, within the framework of the documentary line of specialization, in the undergraduate Audiovisual Arts program at UNAB University. Having in mind classroom practices of teachers involved in the documentary filmmaking modules and, making a review about the state of the art on the theories and the different methods of writing for documentary, certain methodological problems related to the writing process, and recurrent in the specialization line in documentary, are identified; reason why, the aim of the research in the classroom, which is part of the present text, is to establish a method strategy that contributes to the creative process for the design and writing of documentary works in the classroom.
\end{abstract}

Keywords: Film, documentary, script, methodology, textual genres.

AUTORES

René Palomino Rodríguez

Correo electrónico: rpalomino@unab.edu.co

Yulenis Stella Caballero Mier

Correo electrónico: ycaballero9@unab.edu.co

Frank Alexander Rodríguez

Correo electrónico: frodriguez757@unab.edu.co

Docentes de la Facultad de Ciencias sociales, humanidades y artes

Recibido: 5 de diciembre 2017

Universidad Autónoma de Bucaramanga, Bucaramanga, Colombia.

Aprobado: 9 de mayo 2018 


\section{INTRODUCCIÓN}

Desde el año 2012, un poco más de 200 estudiantes han cursado la profundización documental al final de su pregrado; en este marco, se han desarrollado alrededor de 50 proyectos documentales, algunos de ellos con exhibición o circulación nacional e internacional. sSin embargo, se evidenció que la metodología de escritura durante el proceso de diseño y realización de los proyectos, en algunos casos, no contemplaba la reflexión frente a modalidades y formas inherentes al género, desconociendo parte de la evolución expresiva del cine documental y de sus estéticas más contemporáneas. Como resultado, se observa la utilización de formas documentales canónicas, agotadas o subvaloradas en escenarios de intercambio de obras documentales, como festivales o muestras especializadas del género, algo paradójico en obras académicas documentales, que suponen un mínimo de problematización y experimentación acerca de su planteamiento formal y estético.

El objetivo principal de la profundización es brindar al estudiante herramientas conceptuales y metodológicas que le permitan desarrollar un proyecto creativo, apropiando las herramientas del lenguaje audiovisual, en este caso, mediado por la forma documental para construir representaciones del mundo real. En la Figura 1 , se aprecian los diferentes elementos que se contemplan en el proceso creativo.

A partir de este esquema, la obra audiovisual puede entenderse como un discurso sincrético y multimodal. Sincrético, porque está constituido por diversos sistemas de significación y comunicación que, en conjunto, corresponden a un mismo proceso de percepción por parte del destinatario (espectador), quien entra en contacto con la obra fílmica. Por su parte, es multimodal porque el objeto, la obra como bien de intercambio cultural, está constituido por diversos sistemas de comunicación y significación que afectan de manera diferente los sentidos del cuerpo que entra en contacto con ella.

Las obras documentales audiovisuales, en general, remiten a hechos o personajes del mundo real, sin considerar si el personaje aún existe o que los hechos hagan parte del pasado. Este "asunto" o "tema" del que se quiere construir una representación conforma el contenido de la obra, lo que obliga al autor a tener un conocimiento profundo sobre la cuestión o, si no posee este conocimiento, a diseñar una estrategia. De la mirada sobre ese contenido, surge el punto de vista del autor sobre aquello que busca representar.

El proceso creativo comienza cuando el autor se cuestiona acerca de la forma que tendrá su obra y cómo la percibirá el público; es decir, cuando se pregunta cómo construir la representación. Este proceso se denomina cinematografización ${ }^{1}$, proceso en el que el autor debe dominar los códigos del lenguaje audiovisual que constituirán los efectos de sentido que expresarán la obra. Por ejemplo, los códigos que determinan el género cinematográfico, si una pelícu-

\footnotetext{
$1 \quad$ El concepto de cinematografización es explicado por Desiderio Blanco (2003) con relación a la función semiótica del discurso fílmico, al proceso de percepción en el que los códigos fílmicos generan efectos de sentido diferentes a otro tipo de códigos. El texto fílmico como tal logra transmitir sensaciones en el observador (por su capacidad de imitar el mundo que busca representar), al respecto el autor plantea algunos ejemplos: "[...] resulta claro que la forma de cualquier código -el gesto, por ejemplo- adquiere un aspecto, un matiz diferente al inscribirse en un código icónico o en un código verbal. Es decir, un gesto "visto", una pelea "vista", no producen exactamente, los mismos efectos de sentido que un gesto o una pelea "leídos", aunque en ambos casos mantengan su autonomía como códigos. Y la razón de ese contagio reside en el hecho, ya señalado, de que tales códigos constituyen el plano de contenido de otros códigos específicamente cinematográficos, que los invisten de ciertos rasgos específicos, como por ejemplo la iconicidad. Y es en ese sentido en el que se puede seguir hablando de "cinematografización"”' (Blanco, 2003,p.45)
} 
la es de cine negro, responden a un código temático que determina su puesta en escena. Estos códigos pueden alterarse o subvertirse por lo que algunos autores contemporáneos no etiquetan su cine ni como obra de ficción ni como documental.

Lo expuesto anteriormente es una síntesis de la estructura general que determina el proceso creativo en el aula, al interior de la profundización en documental. Esta requiere por parte de los estudiantes, tanto de un ejercicio constante de escritura, como de la apropiación de diversos dispositivos para la escritura de documentales.

A partir de la observación de los procesos de diseño y desarrollo de los proyectos audiovisuales en el aula, se evidenciaron vacíos conceptuales y metodológicos en los estudiantes, entre ellos:

a) Persiste una confusión entre los géneros textuales propios del quehacer audiovisual y no se entienden las formas y el objeto de piezas básicas que describen una obra: la sinopsis, el argumento, el tratamiento audiovisual, entre otros.

b) En algunos procesos se cuestiona la pertinencia de la escritura del guion para el diseño de un documental. Cuando no se cuestiona el proceso de escritura, se plantean alcances temáticos y estéticos que no se reflejan en la calidad final de la obra audiovisual.

c) A pesar de que algunas de estas obras han tenido reconocimientos en distintos ámbitos, los aportes creativos en relación con las formas de representación documental, en general, no han sido muy diferentes al resto de proyectos que se han desarrollado en semestres inferiores.

Por lo anterior, se hace necesaria la implementación de una metodología para el proceso de escritura de proyectos documentales, que derivan (y se involucran) en el proceso de diseño y construcción de obras cinematográficas de carácter documental, para solventar los vacíos metodológicos que dificultan los procesos de investigación-creación en función de los documentales en construcción en el aula.

\section{METODOLOGÍA}

Gouvêa Pimentel (2009) describe la metodología como "una construcción conceptual, elaborada por la intervención del método. Podemos decir que es una espiral de conjunción de métodos, unidos a la innovación de acciones creadoras de nuevos métodos, que a su vez se integrarán a nuevas metodologías, y así sucesivamente" (p.39). Desde esta perspectiva, el proceso metodológico, aunque deriva del método científico, en el caso de las ciencias humanas no siempre se cumplen los pasos del método, ni tampoco en un orden establecido. Podría afirmarse que cada obra conlleva su propio desarrollo metodológico. A este respecto, la autora afirma que, aunque todo método se basa en un sentido del orden, en el caso de las artes el orden que más interesa es el generativo, ligado al proceso de creación: una suerte de hechos y eventos en los cuales la creatividad dicta el rumbo del desdoblamiento, por ser ella la piedra fundamental del proceso (p.38).

A partir de estos planteamientos, se asume el proceso metodológico como una suerte de ruta susceptible de ser recorrida de manera distinta por parte del autor/estudiante. El objetivo es desarrollar una herramienta pedagógica que, si bien contribuye a aclarar las dudas y los vacíos conceptuales y metodológicos evidenciados en los estudiantes e inclusive en los docentes, se busca evitar que se establezca como un proceso cerrado, como una camisa de fuerza que constriña el proceso creativo de cada proyecto. 
Una vez detectados ciertos vacíos conceptuales durante el proceso de diseño de los proyectos documentales, el primer paso metodológico fue la construcción de un estado del arte sobre el proceso del guion para documental. Se tuvo en cuenta que la escritura para el proyecto, denominada "guion", consiste en prever una serie de factores inherentes a la complejidad del registro de situaciones reales, universo en el que no opera ninguna forma de control sobre las situaciones (como sí ocurre en el rodaje de ficción). En el contexto de la investigación y diseño del documental, la escritura adopta diversas formas, como notas personales, notas de investigación o ensayos, donde se alojan datos y percepciones del entorno o sobre el "otro" (el sujeto investigado) e, incluso, propuestas narrativas que describen una estructura inicial de la película y pueden identificar bloques o líneas narrativas que estarán presentes en la narración.

El requerimiento de un texto en donde se describa el proyecto y se prevean diversos aspectos de lo que puede ocurrir en un rodaje documental, aunque decisión exclusiva del director y su método de construcción de la obra, es una herramienta de comunicación del proyecto, al menos para que el resto del equipo técnico comprenda la idea. La escritura es la herramienta básica para que el director pueda comunicar el proceso creativo de su proyecto, o también para ser usada en otras etapas del proceso que tienen que ver con escenarios inherentes a la forma en que se finalizan y distribuyen los proyectos. eEl guion, o "texto", sirve para que el productor, el canal, u otra institución, dependiendo de cada caso, tenga cierta certeza sobre la forma y las características que tendrá la película.

El segundo paso de la metodología fue indagar sobre los géneros textuales propios del quehacer audiovisual que se utilizan durante el desarrollo de los proyectos de aula. El estudio de los géneros tex- tuales nace con la lingüística textual alemana hacia 1960; al respecto, Alexopoulou (2011) describe la pertinencia del estudio de las formas textuales:

El texto, en tanto producto de la actuación lingüística y de la interacción social, presenta una enorme diversidad de producciones múltiples y variadas, creadas en los diferentes ámbitos de uso y con las que los hablantes entran en contacto en su vida cotidiana. Esta realidad generó la necesidad de elaborar sistemas de clasificación con la intención de abarcar todos los textos posibles. Es así que una de las líneas de investigación de la LT se orientó hacia la búsqueda de un sistema de ordenamiento de los tipos de texto con el objetivo de definir los géneros, clasificarlos y construir una tipología a fin de desentrañar la naturaleza de las estructuras textuales (p.98).

En cuanto al origen del género, la autora indica que se remonta a la retórica clásica y que un autor contemporáneo, Mijail Bajtín, a mediados del siglo XX, formula la teoría de los géneros discursivos, argumentando que el género como tal es un "conjunto de enunciados relativamente estable ligado a una esfera social determinada" (p.101). Para Alexopoulou y otros autores, la concepción bajtiniana del lenguaje como objeto ideológico-social analiza el contexto o el campo en el que se dan los intercambios discursivos. Por ello, describe una relación entre las esferas sociales bajtinianas y los ámbitos de uso de los textos según el Marco común de referencia para las lenguas: aprendizaje, enseñanza y evaluación (MCER), que son los siguientes:

a) El ámbito público: hace referencia a todo lo relacionado con la interacción social corriente.

b) El ámbito personal: se refiere a las relaciones familiares y las prácticas sociales individuales.

c) El ámbito profesional: incluye todo lo relativo a las actividades y relaciones de una persona en el ejercicio de su profesión. 
d) El ámbito educativo: tiene que ver con el contexto de aprendizaje o formación (generalmente de carácter institucional), donde el objetivo consiste en la adquisición de conocimientos o destrezas específicas. (MCER, citado por Alexopoulou, 2011, pp.101-102).

Según la teoría de los géneros discursivos, las formas textuales que describen proyectos audiovisuales estarían en el ámbito profesional y, en algunos casos (como en este), en el ámbito educativo. Una de las formas más recurrentes es el guion que, como género, pertenece a muchos otros ámbitos, no únicamente al audiovisual. El guion es utilizado en teatro y otras manifestaciones artísticas, incluso en discursos pedagógicos y publicitarios, donde adquiere diversidad de formas. Cuando se habla de guion para ficción hay unas características canónicas que hacen que su forma sea más homogénea, aunque estas características son subvertidas por algunos autores.

En el caso del guion de documental, el texto puede conformarse de varias formas: puede ser un guion que simule las características del texto para ficción; pueden ser notas de campo, similares a las formas de registro de trabajo de campo propias de la investigación etnográfica; o también pueden adquirir una forma poética, entre muchas otras. Por esta diversidad de formas, se hace necesario una revisión de los modos de escritura para documental utilizadas por autores de cine de no ficción, sean nacionales o extranjeros, que hayan reflexionado acerca de su proceso creativo con relación a la escritura.

A partir de esta revisión teórica y conceptual, tanto acerca de los géneros textuales que influyen en el proceso de escritura, como de los distintos imaginarios y las formas en que asume la escritura para documental, se consolida un manual como herramienta pedagógica para el aula. En este texto didáctico el estudiante podrá aclarar ciertos vacíos acerca de las características y la función de los diversos textos que describen un proyecto audiovisual documental, así como de la relación entre el proceso creativo y la escritura. Esta última acompaña todo el desarrollo de la obra audiovisual, desde su concepción como idea hasta su finalización en la sala de montaje.

\section{LA NECESIDAD DE UNA HERRAMIENTA PEDAGÓGICA QUE DIALOGUE SOBRE POSIBLES FORMAS METODOLÓGICAS PARA EL DOCUMENTAL}

Observando la literatura consultada por docentes y estudiantes en el marco de la profundización documental, se pueden encontrar teorías acerca de la forma y características formales de los modos de representación documental, así como algunos textos que indican el proceso creativo para consolidar la idea para una película. En estos procesos, al igual que en otras escuelas artísticas, el análisis referencial es un paso metodológico obligado para poder analizar las formas de representación documental en obras preexistentes. Sin embargo, no se encontró literatura acerca de las características de las distintas piezas textuales que conforman un proyecto cinematográfico documental, ni es fácil hallar información acerca de la escritura y reescritura del guion a lo largo del proceso creativo. Es decir, hay muchos manuales sobre el guion en general, casi todos para ficción, pero ninguno de ellos es claro en afirmar que, en la mayoría de los casos, el guion se reescribe más de una docena de veces. Esa parte artesanal de la escritura se desconoce porque son procesos que cada autor resuelve de forma distinta, dependiendo también de las características de cada proyecto.

Además de la experiencia en el aula en el desarrollo de proyectos documentales, tanto con los estudiantes de documental de tercer semestre como con los de último año, los hallazgos conceptuales y metodológicos en relación al proceso de escritura 
encontrados en el estado del arte a evidencian que las carencias y vacíos conceptuales y metodológicos son producto de diversos factores, entre ellos, ausencia de literatura específica sobre el tema o confusión entre las piezas textuales entre ficción y documental. Esta distinción, que no resulta problemática para un autor consagrado, sí lo es para un estudiante que enfrenta por primera vez la escritura y justificación de un proyecto documental. Otro factor que confunde al estudiante, en cuanto a la forma de la escritura, consiste en que en la metodología se emplean formas de la investigación en campo del enfoque cualitativo de investigación ${ }^{2}$, como entrevistas semiestructuradas, notas de campo, registro fotográfico y audiovisual del campo. Sus participantes, sin embargo, no son conscientes del objeto de estas formas en la metodología que, en algunos casos, constituyen el insumo del guion documental e inciden en su forma.

\section{EL MANUAL COMO HERRAMIENTA PEDAGÓGICA}

Una vez detectados los problemas metodológicos y sus posibles causas, se decide construir un manual de consulta para estudiantes y docentes en donde se clarifiquen los vacíos conceptuales y metodológicos expuestos anteriormente. Este texto didáctico no pretende constituir una receta para el diseño de proyectos documentales, sino que pretende solucionar inquietudes y abrir posibles caminos para estudiantes y docentes durante su proceso creativo. Tampoco busca establecer un único camino metodológico entre un paso y otro. La ruta puede ser diseñada por cada autor y, aunque parezca pro- blemático, es también lo que enriquece en esencia los procesos creativos.

El texto aclara conceptos y formas metodológicas para el trabajo en cada una de las etapas del proceso generativo de un proyecto audiovisual documental. La estrategia pedagógica pretende que el manual se utilice durante las dos fases de la profundización documental, tanto en la etapa de diseño de proyecto, como en la de realización. De esta forma, los estudiantes y docentes contarán con una herramienta que les permite expresarse con cierta fundamentación acerca de conceptos, métodos y formas documentales que les facilitarán la exploración, solución de problemas y las consultas a otras fuentes durante el proceso de construcción de sus propios proyectos en el aula. Cada proyecto tiene una etapa de gestación y realización de dos a tres semestres.

En este sentido, una de etapa que se ha detectado como crítica es la mitad del proceso, en la profundización, cuando llega el momento de evaluar después de un primer semestre de trabajo de diseño, si el proyecto está listo o no. En la mayoría de los casos, esta evaluación termina siendo subjetiva, por lo que el manual contiene criterios y herramientas que facilitan la evaluación del estado de los proyectos, criterios que no siempre son claros para estudiantes y docentes.

Al final del proceso se evaluará si esta propuesta de intervención en el aula contribuyó al mejoramiento de los procesos creativos y si el resultado de las obras audiovisuales documentales evolucionó en relación a

\footnotetext{
$2 \quad$ A propósito de los objetos de estudio del paradigma cualitativo (lo que en algunos casos evidencia la pertinencia de dispositivos metodológicos cualitativos en el cine documental), Rodríguez, Gil y García sostienen que este enfoque: "estudia la realidad en su contexto natural, tal y como sucede, intentando sacar sentido de, o interpretar los fenómenos de acuerdo con los significados que tienen para las personas implicadas. La investigación cualitativa implica la utilización y recogida de una gran variedad de materiales -entrevista, experiencia personal, historias de vida, observaciones, textos históricos, imágenes, sonidos- que describen la rutina y las situaciones problemáticas y los significados en la vida de las personas" (Rodríguez, Gil y García, 1996, p.32)
} 
nuevas propuestas estéticas y aportes a las formas de representación documental. Uno de los indicadores será que las obras diseñadas y realizadas en el marco de este proyecto circulen, finalmente, como bienes de intercambio cultural en encuentros, festivales y, en general, espacios donde se mida la calidad de las formas documentales en cuanto a su pertinencia, desarrollo técnico, temático y aportes estéticos.

\section{ASPECTOS GENERALES QUE ABORDA EL MANUAL}

\section{a) El documental dentro del enfoque cualitativo de investigación}

No siempre los estudiantes comprenden que la acción de observar y registrar un hecho real, que ocurre en un escenario sociocultural, es una de las herramientas metodológicas de la investigación cualitativa, ni que ello se debe a la existencia de una relación histórica entre la antropología, la etnografía y el documental. Las formas de inmersión y registro de fotógrafos y realizadores documentales en el campo, o en ese espacio que denominamos el "mundo real", tienen su origen en las técnicas desarrolladas por los primeros investigadores sociales. Entender que las técnicas documentales no son un hecho aislado y que, consecuentemente, un documental también es una forma de investigación que proviene o coincide con planteamientos como los de la antropología visual, le da luces al autor en formación para que experimente con técnicas metodológicas en relación con la forma del proyecto y el tema que investiga, ya sea para que profundice en estas técnicas o las cuestione, como han hecho otros autores.

\section{b) Imaginarios del guion documental}

El término imaginarios hace referencia a distintas posturas conceptuales y metodológicas que pueden tener un origen histórico, ya incluso desde los pioneros del género, en los que la idea de un texto preexis- tente, definido en el ámbito cinematográfico como guion, parece no estar suficientemente sustentada, ni en cuanto a su pertinencia, ni a su forma en el cine de documental. Por su parte, sí se halla claramente definida en el proceso creativo del cine de ficción. Autores como Shantal Akerman o José Luis Guerín, quienes no reconocen fronteras entre la ficción y el documental, consideran que el cine es una expresión que se alimenta y se complementa con formas y dispositivos provenientes de ambos géneros.

\section{Ausencia de guion}

Un primer imaginario alrededor de la escritura documental es que se puede prescindir de ella. Así se expone en un reciente texto de Puccini (2015), al hablar de la proliferación contemporánea del cine directo (observacional) entre las nuevas generaciones, por creer que es un cine que puede hacerse de manera más fácil, sin ningún texto presupuesto:

Sospecho que existe alguna otra razón para su popularidad, este documental parece exigir menos trabajo que las formas pasadas del género. Aparentemente, usted no necesita hacer ninguna investigación. Usted no necesita escribir esos guiones aburridos ni narraciones tediosas. Usted no necesita preocuparse por ninguna planificación previa, solamente sale y filma. (Rosenthal citado en Puccini, 2015).

Autores como Jean Luc Godard o el brasilero Eduardo Coutinho han experimentado acerca del trabajo sin guion, o con otras formas de escritura. Sin embargo, esta ruta metodológica se sustenta en el dominio del autor frente a las formas de representación y en el conocimiento que posee sobre aquello que está representando. No es en absoluto una ausencia total de guion, sino que este (sean notas, apuntes, fragmentos de entrevista o guion de montaje) aparece después, durante el proceso. 


\section{El guion como método infalible}

En este sentido, autores como Patricio Guzmán y Michel Rabinger han desarrollado ideas y conceptos propios sobre el proceso de construcción del texto o guion de documental. Incluso, han hecho taxonomías similares sobre posibles estructuras narrativas para el documental, entre ellas:

- Elegir un personaje.

- Elegir un acontecimiento.

- Elegir una situación concreta.

- Hacer un viaje.

- Volver al punto de partida.

Gran parte de los documentales que circulan como bienes de intercambio cultural obedecen a alguna de estas estructuras. De hecho, cada una de estas situaciones puede predeterminar ciertas formas de plantear el discurso o modo de representación, en términos de Nicholls. Al elegir un personaje, puede surgir cierta interacción en el relato; en cuanto al acontecimiento o la situación concreta. Por ello, casi que se hace necesario un registro observacional con la cámara frente al hecho que se pretende registrar. Al narrar un recorrido o un viaje se puede anticipar una exposición por parte del autor o los personajes. En cuanto al volver a un punto de partida, esta ciclicidad (presente también en la literatura, en autores como Borges o García Márquez) puede incluir cualquiera de los modos anteriores, pero presupone un tono ensayístico o poético, en que el autor argumenta o representa el cierre del ciclo, a partir de las posibilidades expresivas fílmicas.

Sin embargo, el guion como tal no resuelve todos los problemas creativos, ni los imprevistos que suelen presentarse en el registro de la realidad. De hecho, su estandarización en documentales institucionales, o en documentales para la televisión o la web, ha ocasionado que se pierda la voz del autor y que se diluya la forma documental hacia otras formas del discurso periodístico.

\section{Ese espacio difuso entre el guion y la película}

Debido a lo anterior, autores que trazaron un método claro de escritura, como Patricio Guzmán, asumen el carácter orgánico del guion, a partir de su propia experiencia. Entre el texto y la película surge un espacio invisible, donde se resuelve la forma que tendrá la obra y que diferencia a un documental de autor de un reportaje, de un documental para televisión o de un documental institucional.

El documental es, entonces, la resultante entre lo predecible y lo impredecible. En esa frontera el género ha encontrado su mayor riqueza, porque cada película se convierte en un objeto de estudio, cada autor idea de manera creativa y orgánica la forma de resolver lo imprevisible, ya sea desde la experimentación estética y narrativa, o ya sea actualizando algunas formas canónicas del género y otorgándoles una nueva apariencia.

El tercer imaginario sería, entonces, la idea de que la película documental resulta no únicamente del guion, sino de un proceso a partir de este, hacia una especie de recorrido de experimentación y creatividad. El guion no cumple una función de guía inmóvil, sino que se considera como la puerta de entrada a un espacio de decisiones y reflexiones, que van tomando formas en situaciones, escenas, o líneas narrativas que deben ser susceptibles de cambiar o desaparecer al entrar en contacto con el registro de lo real.

\section{c) Géneros textuales propios del quehacer audiovisual}

En el ámbito de diversas disciplinas se hallan diferentes grupos y tipos de textos. Por ejemplo, en el 
ámbito legislativo, estarían todos los tipos de texto que describen las diferentes leyes, de donde se alimentan otros textos, como sentencias y demandas que soportan y describen los procesos jurídicos; en el ámbito médico estarían los diferentes textos que contribuyen a describir la situación de los procesos médicos. En el caso del quehacer audiovisual, en el ámbito laboral hay diferentes tipos de texto, además del guion, que contribuyen a la descripción de ideas e intenciones de un proyecto en desarrollo. Se pretende que el manual arroje luces acerca de la forma y función de este grupo de textos, que el estudiante puede hallar en el ámbito universitario y profesional. Estos textos, conforman el grupo de géneros textuales propios del oficio audiovisual, evidencian el proceso creativo y contribuyen al diseño y materialización de las ideas. Entre ellos, puede mencionarse la sinopsis, el tratamiento audiovisual y la nota de dirección. Cada uno de estos textos varía según los requerimientos del destinatario, ya sea un proyecto académico, como en este caso, ya sea un proyecto que concursa en un festival, o un proyecto que se presenta ante un canal o un grupo de inversores. A su vez, estos textos provienen y heredan características similares de otras formas textuales, predecesoras en manifestaciones artísticas, como el teatro y la literatura.

\section{CONCLUSIÓN}

Para que el estudiante vaya más allá de resolver la creación de una película documental (cuestión que ya viene resolviendo en etapas anteriores del pregrado), se debe establecer una metodología de aula que permita el desarrollo de documentales de calidad temática, estética y narrativa y que, además, cuestione a los autores en formación acerca de la pertinencia de sus obras y del aporte de estas al cine documental. El proceso creativo exige un cuestionamiento continuo acerca de diversos aspectos: lo técnico y lo estético, aspectos que se han abordado anteriormente, pero también el aspecto ético. En este, el autor se pregunta por la razón de ser de las obras documentales y por las implicaciones de su inmersión y relación con el grupo social que ha decidido intervenir o con el entorno en donde su obra circulará.

En este encuentro entre diferentes formas de ver el mundo que conforman el campo de trabajo (la del estudiante inexperto y la de los sujetos y su entorno) , surgen intercambios y acuerdos que influyen en ambos participantes. En algunos casos, el joven documentalista es desbordado por el tema y promete más de lo que puede cumplir o resulta manipulado por los sujetos investigados. En otros casos, es el documentalista quien utiliza a los sujetos y sus desgracias como publicidad para su obra, fenómeno que el director argentino Luis Puenzo denominó "pornomiseria".

La preocupación sobre el objeto del cine documental ha estado presente desde los autores pioneros del género. Al respecto, Paula Ortiz (2008) describe la visión de Flaherty y Grierson:

Flaherty se situaba en una cierta posición roussoniana donde el conocimiento del otro, del extranjero, del hombre sin decadencias, favorecería una mutua comprensión de los pueblos. Por su parte, Grierson reivindicaba una mayor responsabilidad de los narradores documentalistas, exigiendo una posición más lúcida y madura con el espíritu contemporáneo. Para ello, pedía la búsqueda de una nueva mirada creativa y poética que devolviera un reflejo enamorado de la realidad. ( $p .839$ ).

Robert Flaherty tenía una visión positiva acerca del cine documental, pensaba que un sujeto o un grupo de sujetos podrían entender y comprender a los otros a partir de la observación de sus formas de vida. Por otro lado, Jhon Grierson, quien desarrolló 
la escuela británica de documentalistas, buscaba un espíritu crítico y lúcido entre sus discípulos, para que todo el tiempo se preguntaran por las formas de representación y por la evolución del lenguaje del cine documental. Ambos planteamientos son similares a los expuestos en el objeto de la presente investigación y buscan fortalecer a las nuevas generaciones de documentalistas para que construyan representa-

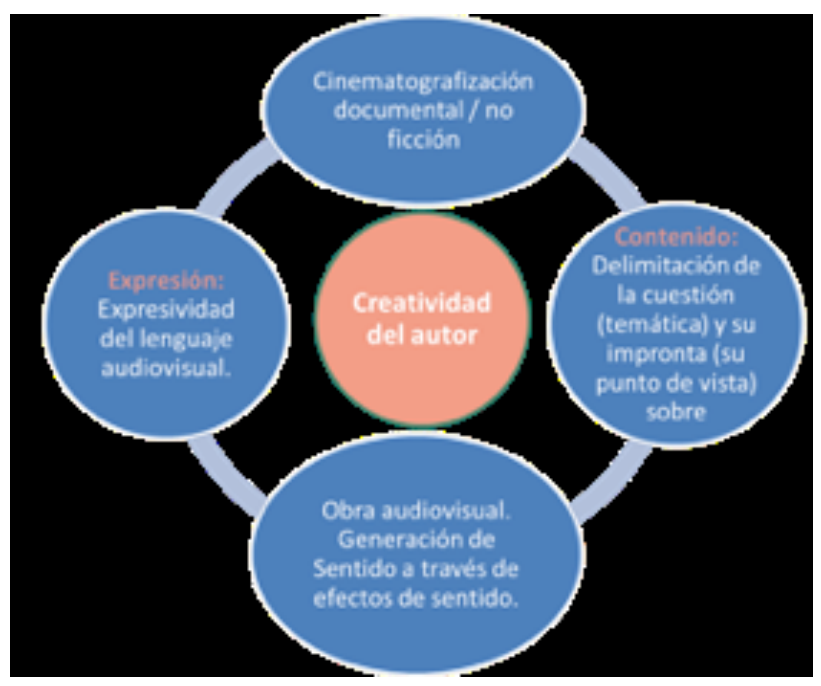

ciones que hablen desde la región oriental de Colombia y que puedan dialogar con el cine documental de primer orden en el mundo.

Figura I. Esquema del proceso creativo de una obra audiovisual, a partir del esquema de L. Hjelmslev sobre la función semiótica contenido-expresión. La obra se asume como un signo, el significado es el contenido y el significante la expresión cinematográfica que construye los efectos de sentido, para que el contenido sea percibido por el destinatario, el espectador de la obra. En el caso de las obras audiovisuales, entre el significante y el significado se establecen diferentes planos de construcción de sentido desde las figuras que constituyen el filme, acciones y movimientos que constituyen programas narrativos, hasta el sistema axiológico que subyace en el relato y que finalmente, determina su contenido.

\section{REFERENCIAS}

Alexopoulou, A. (2011). El enfoque basado en los géneros textuales y la evaluación de la competencia discursiva. Del texto a la lengua: la aplicación de los textos a la enseñanza-aprendizaje del español L2-LE. Actas del XXI Congreso Internacional de ASELE. Salamanca: Imprenta Kadmos.

Blanco, D. (2003). Semiótica del texto fílmico. Lima: Fondo desarrollo editorial- Universidad de Lima.

Nicholls, B. (1997). La representación de la realidad, cuestiones y conceptos sobre el documental. BarceIona: Editorial Paidós.

Ortiz, P. (2008). Flaherty, Griegson e Ivens: la exploración del mundo lejano y el cercano. Fundamentos estético-éticos en los orígenes del género documental. Artigrama, 23, 823-839. Recuperado el 16 de junio de 2016 de https://www.unizar.es/artigrama/ pdf/23/3varia/20.pdf

Pimentel, L. G. (2009). Metodologías de la enseñanza de arte: algunos puntos para debatir. Revista Educación y Pedagogía,li. 21n (55), .31-42.

Puccini, S. (2015). Guion de documentales: de la preproducción a la producción. Ciudad Autónoma de Buenos Aires: La marca editora.

Rodríguez, G., Gil, J., García, E. (1996). Metodología de la investigación cualitativa. Granada: Ediciones Aljibe. 